Int. J. Dev. Biol. 48: 679-682 (2004)

doi: $10.1387 / \mathrm{ijdb} .041862 \mathrm{~ns}$

Short Communication

\title{
Identification of new modes of Dd-STATa regulation of gene expression in Dictyostelium by in situ hybridisation
}

\author{
NAO SHIMADA ${ }^{1}$, MINEKO MAEDA ${ }^{2}$, HIDEKO URUSHIHARA ${ }^{3}$ and TAKEFUMI KAWATA*,1 \\ ${ }^{1}$ Department of Biology, Faculty of Science, Toho University, Funabashi, Chiba, Japan, ${ }^{2}$ Department of Biology, Graduate School of \\ Science, Osaka University, Toyonaka, Osaka, Japan and ${ }^{3}$ Biological Sciences, University of Tsukuba, Ibaraki, Japan
}

\begin{abstract}
Signal Transducers and Activators of Transcription (STATs) are transcription factors which lie at the end of cytokine and growth signal transduction pathways. Dictyostelium Dd-STATa is a functional homologue of metazoan STATs. It is activated by cAMP and, at the slug stage, it translocates into the nuclei of the tip cells, which are a subset of the anterior, prestalk $A$ (pstA) cells. Here we searched for novel Dd-STATa regulated genes by in situ hybridisation. A set of 54 cDNA clones whose gene expression patterns are known to be prestalk-specific (Maeda et al., 2003), were chosen as probes and we compared their expression patterns in parental and Dd-STATa-null strains. We identified 13 genes which are candidates for direct induction by Dd-STATa. In the parental strain, most of these genes are expressed in the cone shaped mass of pstAB cells which is located within the prestalk region. These cDNAs show little or no expression in the Dd-STATa-null strain. This contrasts markedly with the paradigmatic ecmB gene which is expressed in pstAB cells in parental cells, but which is expressed throughout the prestalk zone in the Dd-STATa-null strain. We also identified several genes which are normally expressed in pstA cells, or throughout the prestalk region, but whose expression is markedly down-regulated in the null mutant. Again, this contrasts with markers derived from the paradigmatic, ecmA gene which are expressed normally in the $D d$ STATa-null strain. The identification of these novel genes provides valuable tools to investigate the role of Dd-STATa.
\end{abstract}

KEY WORDS: in situ hybridisation, transcriptional regulation, Dictyostelium, STAT transcription factor

STATs are transcription factors which lie at the end of cytokine or growth factor signal transduction pathway. They play a role in many important biological phenomena and are evolutionarily conserved among multicellular eukaryotic organisms (Darnell, 1997; O'Shea et al., 2002). Recently, it has also been reported that STATs are involving in regulating morphogenetic cell movement (Hou et al., 2002). Thus, it is of importance to know which gene classes are regulated by STATs. For this purpose we used the cellular slime mould Dictyostelium, the simplest eukaryote having STAT proteins, as a model organism.

In Dictyostelium, four STAT genes, Dd-STATa, $b, c$ and $d$ have been identified (Kawata et al., 1997; Mohanty et al., 1999; Fukuzawa et al., 2001; Zhukovskaya et al., 2003; Abe and Williams, unpublished). Dd-STATa is necessary for efficient chemotactic cell movement at the aggregation stage and morphogenesis after the slug stage (Mohanty et al., 1999), suggesting that Dd-STATa is involved in aggregation and multicellular development by regulating chemotactic cell movement. In the slug tip, Dd-STATa is phosphorylated on a tyrosine residue in response to CAMP signaling and then translocates into nuclei (Kawata et al., 1997; Araki et al., 1998). Therefore, Dd-STATa seems likely to regulate gene expression in the tip region of the slug. In support of this hypothesis, the Dd-STATa-null mutant lacks a subset of the tip cells, does not express the $c u d A$ gene (Fukuzawa and Williams, 2000) in the tip and fails to culminate (Mohanty et al., 1999). Therefore, genes regulated by DdSTATa presumably have important functions during culmination. As STAT signalling networks are known to be evolutionarily conserved, hunting for such genes is of considerable importance. However, ecmB and $c u d A$ are the only target genes of Dd-STATa thus far known (Mohanty et al., 1999; Fukuzawa and Williams, 2000).

Abbreviations used in this paper: ALC, anterior-like cells; Dd, Dictyostelium discoideum; psp, prespore; pst, prestalk; STATs, signal transducers and activators of transcription.

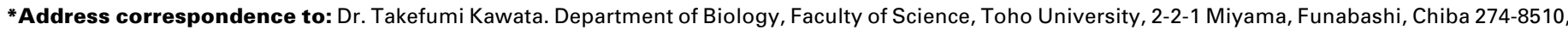
Japan. Fax: +81-47-472-5156. e-mail: tkawata@bio.sci.toho-u.ac.jp
} 
In order to understand the role of Dd-STATa in Dictyostelium, we have searched for Dd-STATa target genes, which are expressed in the anterior tip cells and positively regulated by Dd-STATa protein. In situ hybridisation analyses identified 13 new candidate Dd-STATa target genes. Most of these genes are expressed in the cone shaped mass of prestalk $A B$ cells in the wild-type strain. In addition, several Dd-STATa dependent genes are expressed in the pstA (cells that express the proximal, pstA-specific part of the ecmA promoter) and pstAO cells (cells which express the distal, pstO-specific part and the proximal, pstA-specific part of the ecmA promoter). The role of Dd-STATa is discussed in terms of morphogenetic cell movement via the newly identified target candidate genes.

\section{Results \& Discussion}

Dd-STATa can act as either transcriptional activator or repressor in Dictyostelium (Mohanty et al., 1999; Fukuzawa and Williams, 2000). In order to search for the Dd-STATa target genes, we focused on the prestalk-specific genes whose expressions seemed likely to be positively regulated by $\mathrm{Dd}$ STATa; because the Dd-STATa gene is expressed in the pstA cells at the early slug stage (Fig. 1; Shimada et al., 2004) and Dd-STATa protein is activated by cAMP through the cyclic AMP receptor (cAR1) and then translocates into nucleus of pstA cells (Araki et al., 1998).

Among the 104 ESTs previously analysed by in situ hybridisation, we chose 37 pstA or pstAO, 8 pstAB and 8 late prestalk genes whose expression patterns are very clear (Maeda et al., 2003). Expression patterns of all these genes were analyzed and compared between wild-type $(A \times 2)$ and $D d$ STATa-null strains (Tables I, II). Most of pstA/AO genes were expressed even in the absence of Dd-STATa (Table II), suggesting that Dd-STATa is unlikely to act as a transcriptional activator for these genes. Also, all 8 late "prestalk" genes, which are not expressed at the slug stage but that become expressed during culmination, showed indistinguishable patterns in wild type and Dd-STATa-null strains, This suggests that Dd-STATa is unlikely to be a repressor for these genes at the slug stage.

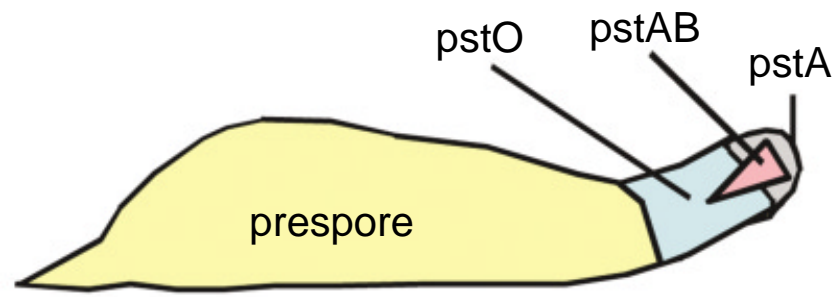

Fig. 1. Schematic illustration of cell types in a Dictyostelium slug. The slugs of Dictyostelium are composed of two basic cell types, prespore (psp) and prestalk (pst) cells. Prestalk cells are further divided into several subtypes, pstA, pstO and pstAB cells, based on the expression of promoter subfragment of ecmA and ecmB genes (Jermynet al., 1996). The pstA cells occupy the front half of the anterior prestalk cells, while the pstO cells occupy the rear half of the pst cells. There are additional prestalk subpopulations, anterior like cells (ALC) and rearguard cells, which are not indicated in this illustration.
TABLE 1

\section{Dd-STATa-INDUCED PRESTALK-SPECIFIC GENES IN DICTYOSTELIUM}

Accession no. cDNA Product

\section{PstA/AO genes}

AU038606 SSL238

AU060824 SLB609

C90144 SS/141

C91531 SSK395

$\begin{array}{lc}\text { Extracellular matrix protein } & 1 \mathrm{e}-05 \\ \text { Extracellular matrix protein St15 } & 0 \\ \text { Acid phosphatase } & 5 \mathrm{e}-22 \\ \text { base-nonspecific/acid RNase } & 1 \mathrm{e}-123 \\ \text { Homologous to Adducin 1 (alpha) head } & 1 \mathrm{e}-52\end{array}$

PstAB genes

AU060958

$S L C 388$

AU060722

$S L B 233$

AU060076

$S L A 128$

AU061846

SLG322

AU038850 SSL558

AU062236 SL/604

C91965

SSC596

Dictyostelium steroid isomerase

$5 e-45$

Arabidopsis dynamin-like protein

$2 \mathrm{e}-42$

Homologous to expansin

$5 e-10$

Carboxypeptidase

$5 e-62$

Homologous to endo-1,4-b-glucanase 2e-76

Aconitate hydratase

0.0

AU074317

SSK348

Unknown

Interestingly, however, we were able to identify 13 new candidate Dd-STATa induced genes. Expressions of five pstA/AO genes, SLF308, SSL238, SLB609, SS/141 and SSK395 were greatly reduced or totally absent in the Dd-STATa-null strain (Fig. $2 \mathrm{~A}, \mathrm{~B}$, Table I). As shown by these results, cells expressing these genes almost overlapped the cell-types of wild type slugs where Dd-STATa is activated (Figs. 1 and 2 A,B and Araki et al., 1998). Thus, the loss of such gene expression suggests that Dd-STATa could serve as a direct activator of these genes. Alternatively, DdSTATa may indirectly activate expressions of the five genes. The fact that the expressions of other prestalk-specific genes, as listed in Table 2, were all expressed even in the absence of Dd-STATa is very striking; thus Dd-STATa appears to be involved in transcriptional regulation in different manners, gene by gene, even in the same cell types.

TABLE 2

\section{Dd-STATa-INDEPENDENT PRESTALK-SPECIFIC GENES USED IN} THIS STUDY

PstA/AO genes

SSM194 SSF823 SSB559 SSG767 SLF535

SSA854 SSM416 SSD873 SLA387 SSA210

SSJ246 SSJ814 SLG775 SSL349 SLF774

SSA358 SLK182 SSH630 SSL853 SSL550

SSB337 SLE474 SSF295 SSM642 SSK232

SSJ314 SSG805 SSK159 SSM184 SSK861

SSF509 SSH475

Not expressed at SSE634 SSD492 SSG357 SSG721 SLH173

slug stage SSD123 SLH752 SLI271 
We also found that all pstAB genes, which we identified previously, are not expressed in the pst $A B$ cells in the mutant slugs (Fig. 2 C,D, Table I). These results again suggest that Dd-STATa might act as a transcriptional activator for these pstAB genes. However, we cannot rule out the possibility that pst $A B$ cells are simply absent from the Dd-STATa-null, because the expression of the $S T:: / a c Z$ marker gene, the paradigmatic pstAB marker, is absent in the Dd-STATanull mutant (Mohanty et al., 1999). Among these pstAB genes, several such as SSL558 are expressed in pstA and pstO cells at the earlier, tipped mounds and standing slug stages (see Fig. 2D yellow arrowheads). Expressions of these genes in both pstA and pstO cells was not affected by the absence of Dd-STATa. Therefore, these results indicate that the regulatory mechanism necessary for the expression of these genes in pstA and pstO cells differs from that in pstAB cells.

In conclusion, we have identified 13 new candidate Dd-STATa-regulated genes by in situ hybridisation. Eight are pstAB genes and five are pstA/AO genes. It remains to be determined whether Dd-STATa directs their expression directly or indirectly. A more extensive analysis of these genes should provide useful information with which to understand the molecular mechanism whereby Dd-STATa to controls Dictyostelium pattern formation.

\section{Experimental Procedures}

\section{Cells and growth conditions}

$D$. discoideum Ax2 and Dd-STATa-null cells were cultured axenically in $\mathrm{HL} 5$ medium at $22^{\circ} \mathrm{C}$. Cells of the $D d$-STATa-null strain were kept growing in HL5 supplemented $10 \mu \mathrm{g} / \mathrm{ml}$ blasticidine S (Kaken Pharmaceutical, Tokyo, Japan).

\section{In situ hybridisation}

Whole-mount in situ hybridisation analyses were performed as described previously (Escalante and Loomis, 1995; Maeda et al., 2000; 2003). In this study, Ax2 and Dd-STATa-null cells in an Ax2 background were allowed to develop on filter paper (FILTER PAPER 4A, ADVANTEC, Tokyo, Japan) placed on nonnutrient agar at $22^{\circ} \mathrm{C}$. After fixation, samples were hybridised in microtubes. RNA probes were synthesized using DIG RNA labeling kit (Roche, Germany) using T7 or SP6 RNA polymerase according to the manufacturer's instruction.

\section{Acknowledgements}

This work was partly supported by The Naito Foundation, The Sumitomo Foundation, The Yamada Foundation and Faculty of Science Special Grant for Promoting Scientific Research at Toho University to $T$. Kawata, the Future of the Japan Society for the Promotion of Science to M. Maeda, as a member of the Kuhara's Project (JSPS-RFTFOOL01412) and H.

\section{A}

Ax2

stat $A^{-}$

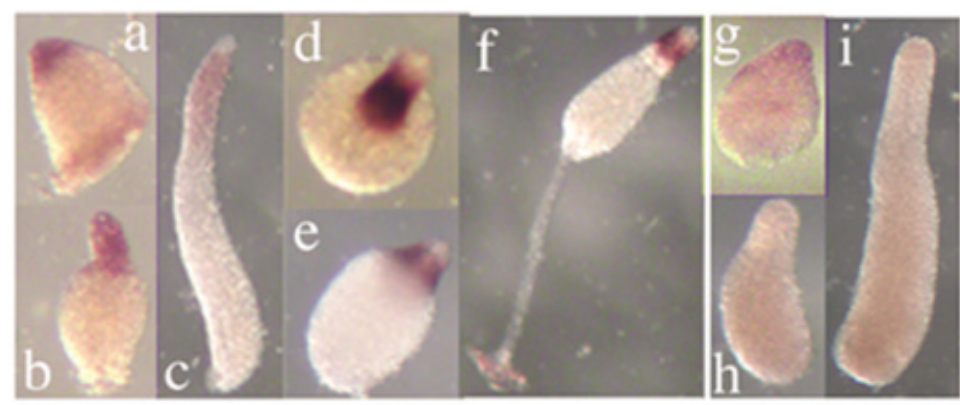

SSL238

B

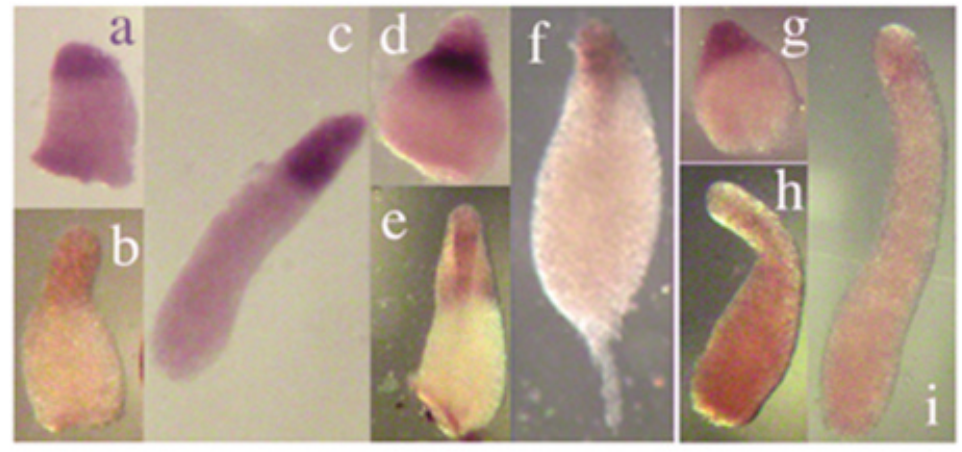

SSK395

C

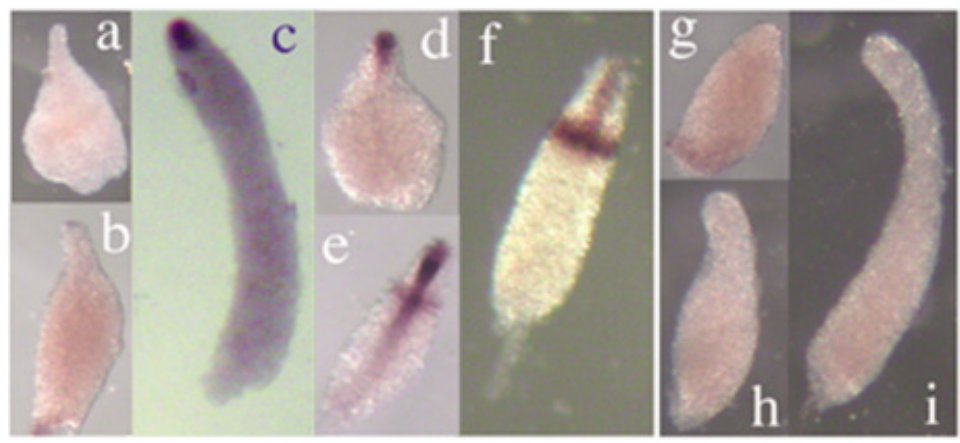

SLC388

D

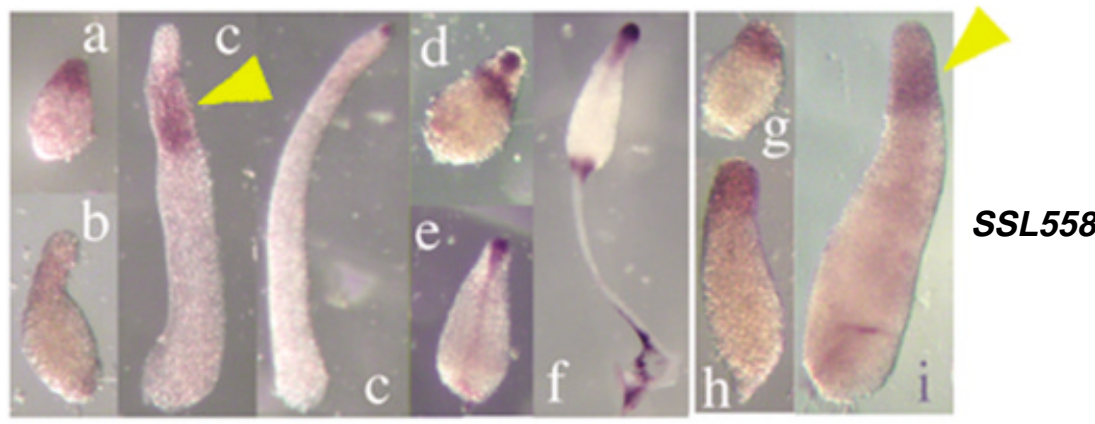

Fig. 2. Expression profiles of several prestalk-specific genes in both wild-type (Ax2) and Dd-STATa-null mutant. (a-d) Spatial expression of (A) SSL238, (B) SSK395, (C) SLC388 and (D) SSL558 genes detected by in situ hybridisation. Photographs show individual developmental stages of Ax2 (a-f), for (a) tipped mound, (b) first finger, (c) slug, (d) Mexican hat, (e,f) early culminants and Dd-STATa-null mutant (g-i), for (g) tipped mound, (h) first finger and (i) slug. 
Urushihara (JSPS-RFTF96L00105) and by a Grant-in-Aid for Scientific Research on Priority Areas C from Ministry of Education, Culture, Sports, Science and Technology of Japanto H. Urushihara (\#12206001). We are grateful to Prof. Jeffrey G. Williams of University of Dundee, UK, for his critical reading of the manuscript and encouragement throughout the entire works.

\section{References}

ARAKI, T., GAMPER, M., EARLY, A., FUKUZAWA, M., ABE, T., KAWATA, T., KIM, E., FIRTEL, R.A. and WILLIAMS, J.G. (1998). Developmentally and spatially regulated activation of a Dictyostelium STAT protein by a serpentine receptor. EMBO. J. 17: 4018-4028.

DARNELL, J.E. (1997). STATs and gene regulation. Science 277: 1630-1635.

ESCALANTE, R. and LOOMIS, W.F. (1995). Whole-mount in situ hybridization of celltype-specific mRNAs in Dictyostelium. Dev. Biol. 43: 262-266.

FUKUZAWA, M. and WILLIAMS, J.G. (2000). Analysis of the promoter of the cudA gene reveals novel mechanisms of Dictyostelium cell type differentiation. Deve/opment 127: 2705-2713.

FUKUZAWA, M., ARAKI, T., ADRIAN, I. and WILLIAMS, J.G. (2001). Tyrosine phosphorylation-independent nuclear translocation of a Dictyostelium STAT in response to DIF signaling. Mol. Cel/7: 779-788.

HOU, S.X., ZHEN, Z., CHEN, X. and PERRIMON, N. (2002). The JAK/STAT pathway in model organisms: Emerging roles in cell movement. Dev. Cel/3: 765-778.

JERMYN, K.A., TRAYNOR, D. and WILLIAMS, J.G. (1996). The initiation of basal disc formation in Dictyostelium discoideum is an early event in culmination. Development 122: 753-760.

KAWATA, T., SHEVCHENKO, A., FUKUZAWA, M., JERMYN, K.A., TOTTY, N.F., ZHUKOVSKAYA, N.V., STERLING, A.E., MANN, M. and WILLIAMS, J.G. (1997). $\mathrm{SH} 2$ signaling in a lower eukaryote: A STAT protein that regulates stalk cell differentiation in Dictyostelium. Cel/89: 909-916.

MAEDA, M., KUWAYAMA, H., YOKOYAMA, M., NISHIO, K., MORIO, T., URUSHIHARA, H., KATHO, M., TANAKA, Y., SAITO, T., OCHIAI, H., TAKEMOTO, K., YASUKAWA, H. and TAKEUCHI, I. (2000). Developmental changes in the spatial expression of genes involved in myosin function in Dictyostelium. Dev. Biol. 43: 114-119.
MAEDA, M., SAKAMOTO, H., MARUO, T., OGIHARA, S., IRANFAR, N., FULLER, D., MORIO, T., URUSHIHARA, H., TANAKA, Y. and LOOMIS, W.F. (2003). Changing patterns of gene expression in prestalk cell subtypes of Dictyostelium recognised by in situ hybridisation with genes from microarray analyses. Eukaryotic Cel/2: 638-645.

MOHANTY, S., JERMYN, K.A., EARLY, A., KAWATA, T., AUBRY, L., CECCARELLI, A., SCHAAP, P., WILLIAMS, J.G. and FIRTEL, R.A. (1999). Evidence that the Dictyostelium Dd-STATa protein is a repressor that regulates commitment to stalk cell differentiation and is also required for efficient chemotaxis. Development 126: 3391-3405.

MORIO, T., URUSHIHARA, H., SAITO, T., UGAWA, Y., MIZUNO, H., YOSHIDA, M., YOSHINO, R., MITRA, B.N., PI, M., SATO, T., TAKEMOTO, K., YASUKAWA, H., WILLIAMS, J., MAEDA, M., TAKEUCHI, I., OCHIAI, H. and TANAKA, Y. (1998). The Dictyostelium developmental cDNA project: generation and analysis of expressed sequence tags from the first-finger stage of development. DNA Res 5: 335-340.

O'SHEA, J.J., GADINA, M. and SCHREIBER, R.D. (2002). Cytokine signaling in 2002: New surprises in the Jak/Stat pathway. Cel/109: S121-S131.

SHIMADA, N., NISHIO, K., MAEDA, M., URUSHIHARA, H. and KAWATA, T. (2004). Extracellular matrix family proteins that are potential targets of DdSTATa in Dictyostelium discoideum. J. Plant Res. (in press)

ZHUKOVSKAYA, N.V., FUKUZAWA, M., TSUJIOKA, M., JERMYN, K.A., KAWATA, T. ABE, T., ZVELEBIL, M. and WILLIAMS, J.G. (2004). Dd-STATb, a Dictyostelium STAT protein with highly aberrant SH2 domain, functions as a regulator of gene expression during growth and early development. Development 131: 447-458.
Received: June 2004 Reviewed by Referees: August 2004 Modified by Authors and Accepted for Publication: August 2004 